\title{
Estudo comparativo do volume de oxigênio máximo entre diferentes modalidades esportivas
}

\author{
Comparative study of the maximum oxygen volume between diferente sports modalities \\ Estudio comparativo del volumen máximo de oxígeno entre diferentes modalidades deportivas
}

Recebido: 20/07/2021 | Revisado: 29/07/2021 | Aceito: 27/08/2021 | Publicado: 29/08/2021

\author{
Maria Williane Batista Magalhães \\ ORCID: https://orcid.org/0000-0003-4359-2504 \\ Centro Universitário Santo Agostinho, Brasil \\ E-mail:mariawillianebatista@gmail.com \\ Ângela Letícia Silva Lima \\ ORCID: https://orcid.org/0000-0001-6772-4722 \\ Centro Universitário Santo Agostinho, Brasil \\ E-mail:angelalslima@gmail.com \\ Andressa Laila Silva Lima \\ ORCID: https://orcid.org/0000-0003-2545-639X \\ Centro Universitário Santo Agostinho, Brasil \\ E-mail: andressaslima@gmail.com \\ Eduardo Kellyton de Oliveira Costa \\ ORCID: https://orcid.org/0000-0002-6232-2967 \\ Centro Universitário Santo Agostinho, Brasil \\ E-mail:eduardo7k@gmial.com \\ Josias Moreira de Castro \\ ORCID: https://orcid.org/0000-0003-3046-0385 \\ Centro Universitário Santo Agostinho, Brasil \\ E-mail:josiasmcastro@gmail.com \\ Lucília da Costa Silva \\ ORCID: https://orcid.org/0000-0001-9386-5684 \\ Centro Universitário Santo Agostinho, Brasil \\ E-mail:luciliafisio@outlook.com \\ Wellington dos Santos Alves \\ ORCID: https://orcid.org/0000-0003-1114-773X \\ Universidade Estadual do Piauí, Brasil \\ E-mail: wellingtonsantos@ccs.uespi.br
}

\begin{abstract}
Resumo
Introdução: O consumo de oxigênio máximo (VO2máx) é a variável que se relaciona com a aptidão cardiorrespiratória e representa a capacidade máxima do organismo em captar, transportar e utilizar o oxigênio pelas células durante o exercício físico. Objetivos: O presente estudo teve como objetivo determinar o $\mathrm{VO}_{2}$ máx através do teste indireto, em estudantes universitários praticantes de atividades esportivas de diferentes modalidades. Metodologia: caracteriza-se como comparativo descritivo, prospectivo, transversal. A amostra foi composta por 14 estudantes praticantes de diferentes modalidades esportivas do Centro Universitário Santo Agostinho na cidade de Teresina, Pi. A coleta de dados foi realizada através do teste de 2400 metros na pista de corrida Parque Potycabana. Resultados: 14 voluntários que atenderam aos critérios de inclusão do estudo 10 eram sexo masculino $(71,4 \%)$ e 4 sexo feminino $(28,6 \%), 8$ voluntários de futsal $(57,1 \%)$ e 6 de handebol $(42,9 \%)$. Foi utilizado o teste de associação Qui-quadrado de associação dos dados qualitativos sociais (idade, sexo e modalidade esportiva), foi também utilizado o teste de comparação de média para os dados quantitativos para os resultados fisiológicos (frequência de treinamento, pressão arterial média inicial, pressão arterial média final, frequência cardíaca, frequência respiratória, saturação, VO2). Conclusão: O estudo comprovou que existe nível de significância entre VO2 máx, aptidão física, sexo, frequência cardíaca média final e tempo de percurso, presente nos 14 atletas avaliados, Não foram encontrados resultados significantes entre as variáveis: modalidade, em comparação ao nível de VO2 máx e idade.
\end{abstract}

Palavras-chave: Aptidão física; Volume de oxigênio; Volume máximo; Esportes.

\begin{abstract}
Introduction: Maximum oxygen consumption ( $\mathrm{VO} 2 \mathrm{max}$ ) is the variable that is related to cardiorespiratory fitness and represents the body's maximum capacity to capture, transport and use oxygen by cells during physical exercise. Objective: This study aimed to determine the VO2max through the indirect test, in university students practicing sports activities of different modalities. Methodology: characterized as descriptive, prospective, transversal comparative. The sample consisted of 14 students practicing different sports at the Centro Universitário Santo Agostinho in the city of
\end{abstract}


Teresina, Pi. Data collection was performed behind the 2400 meter run test on the Parque Potycabana running track. Results: 14 volunteers who met the inclusion criteria of the study 10 were male $(71.4 \%)$ and 4 female $(28.6 \%), 8$ futsal volunteers $(57.1 \%)$ and 6 handball $(42.9 \%)$. The Chi-square association test of association of social qualitative data (age, sex and sport modality) was used, the mean comparison test was also used for quantitative data for physiological results (training frequency, initial mean blood pressure, final mean arterial pressure, heart rate, respiratory rate, saturation, VO2). Conclusion: The study proved that there is a significance level between VO2 max, physical fitness, gender, final average heart rate and time of travel, present in the 14 athletes evaluated. No significant results were found between the variables: modality, compared to the level of VO2 max and age.

Keywords: Physical aptitude; Oxygen volume; Maximum volume; Sports.

\section{Resumen}

Introducción: consumo máximo de oxígeno (VO2max) es la variable que se relaciona con la aptitud cardiorrespiratoria y representa la capacidad máxima del cuerpo para captar, transportar y utilizar oxígeno por las células durante el ejercicio físico. Objetivo: Este study tiene como objetivo determinar el VO2max a través de la prueba indirecta, en estudiantes universitarios que practican actividades deportivas de diferentes modalidades. Metodología: caracterizada como descriptiva, prospectiva, comparativa transversal. La muestra estuvo conformada por 14 estudiantes que practican diferentes deportes en el Centro Universitário Santo Agostinho de la ciudad de Teresina, Pi. Se realizó la de datos se realizó detrás de la prueba de carrera de 2400 metros en la pista de atletismo Parque Potycabana. Resultados: 14 voluntarios que cumplieron los criterios de inclusión del estudio, 10 eran hombres $(71,4 \%)$ y 4 mujeres $(28,6 \%), 8$ voluntarios de fútbol sala $(57,1 \%)$ y 6 de balonmano $(42,9 \%)$. Se utilizó la prueba de asociación Chi-cuadrado de asociación de datos sociales cualitativos (edad, sexo y modalidad deportiva), también se utilizó la prueba de comparación de medias para los datos cuantitativos de los resultados fisiológicos (frecuencia de entrenamiento, presión arterial media inicial, presión arterial media final, frecuencia cardíaca, frecuencia respiratoria, saturación, VO2). Conclusión: El estudio demostró que existe un nivel de significancia entre el VO2 max, condición física, sexo, frecuencia cardíaca promedio final y tiempo de viaje, presente en los 14 deportistas evaluados. No se encontraron resultados significativos entre las variables: modalidad, en comparación con la nivel de VO2 máx. y edad.

Palabras clave: Aptitud física; Volumen de oxígeno; Volumen máximo; Deportes.

\section{Introdução}

O consumo máximo de oxigênio (VO2máx) é definido como a mais alta taxa de oxigênio consumida que extrai, transporta, usa moléculas de oxigênio para realizar um esforço máximo e sua determinação gera informações sobre prática de fornecimento de energia e nível de aptidão física (Alves; Pelissari, 2015).

A determinação desta variável reflete a integração entre o sistema respiratório, cardiovascular e neuromuscular fazendo com que o VO2máx se mostre uma medida fundamental para a prescrição de treinamento. Para que aconteça um treinamento esportivo adequado deve-se ter compreensão de que se trata de um processo sistematizado, envolvendo fatores da organização, da preparação do atleta e a busca da obtenção do melhor resultado (Souza; Vieira, 2016).

A determinação da potência aeróbica tem como indicador da sua capacidade o VO2 máximo, que é um valioso parâmetro preditor de morbidades associadas, além de ser utilizado para acompanhamento e prescrição do treinamento aeróbio em atletas e sedentários, podem ser realizados alguns testes, uns se caracterizam por serem mais complexos e precisos, os testes diretos ou invasivos, outros eficazes e simples, os testes indiretos ou não invasivos, sendo este último, um muito utilizado pela sua efetividade e baixo custo (Corrêa; Moreno, 2018).

As duas medidas conhecidas para saber qual o valor do gasto máximo de oxigênio e aptidão cardiorrespiratória, são as formas diretas e a estimativa indireta, são medida que estão em alta nos campos de pesquisas tanto na clínica, esporte e o valor do rendimento de determinado indivíduo ou atleta, pois seus resultados são confiáveis e indicativos para potência aeróbica (Reis; Júnior, 2018).

Os testes indiretos executados em campo estão na possibilidade em assegurar uma maior especificidade do contexto esportivo, e sua desvantagem está na dificuldade de padronizações e, assim, em garantir sua fidedignidade e validade ao aplicálo em diferentes populações. Ao contrário, a vantagem dos testes indiretos realizados em ergômetros padrões é uma melhor padronização do seu protocolo e controle das variáveis de desempenho, mas como desvantagens apresenta-se a falta de especificidade esportiva (Freitas; Maurício, 2014). 
$\mathrm{O} \mathrm{VO}_{2}$ máx é uma medida importante para se observar a capacidade funcional de cada indivíduo praticante de uma modalidade esportiva, para demonstrar habilidades aeróbicas. Esse teste vai medir a capacidade que o organismo tem de oferecer e utilizar energias. Com essa melhora da capacidade máxima de oxigênio, resultará em um transporte mais eficaz de oxigênio para os músculos, retardando ao máximo a fadiga e aumentando a eficiência dos atletas (Politano; Pereira, 2020).

Compreender essas medidas é de suma importância para ajustar cargas de treinamentos físicos e aptidões cardiorrespiratórias, evitando, com isso, fadiga muscular e morbidades permitindo um acompanhamento do rendimento e dos efeitos dos exercícios sobre a desempenho funcional dos atletas universitários praticantes de futsal e handebol. Assim, esta pesquisa justifica-se ao comparar o $\mathrm{VO}_{2}$ máx entre essas modalidades.

$\mathrm{O}$ presente estudo teve como objetivo determinar o $\mathrm{VO}_{2}$ máx através do teste indireto, em 14 estudantes universitários praticantes de atividades esportivas de diferentes modalidades.

\section{Metodologia}

A pesquisa trata-se de um estudo comparativo descritivo, com a participação de 14 voluntários universitários do sexo masculino e feminino praticantes de diferentes modalidades esportivas. Nenhum deles estavam envolvidos em atividades competitivas, no entanto, eram praticantes de esportes recreativos, sendo eles da atlética do Centro Universitário Santo Agostinho com as modalidades de handebol e futsal (71,4\% masculino, 28,6\% feminino). A coleta de dados foi realizada na pista de corrida do Parque Potycabana em Teresina, Piauí, após aprovação pelo comitê de ética e pesquisa, CEP 2.038.914.

Todos os participantes do estudo concordaram em assinar o Termo de Consentimento Livre e Esclarecido, conforme a Resolução do Conselho Nacional de Saúde 466/12. O trabalho teve como critérios de inclusão: indivíduos universitários praticantes de diferentes modalidades esportivas (futebol e handebol), idade igual ou superior a 18 anos, não tivessem cardiopatias, histórico de pneumopatias nos últimos 12 meses, e não treinassem com frequência mínima de três vezes por semana.

Foram excluídos do presente estudo os atletas profissionais, aqueles cujo tenham diagnóstico de asma, doença pulmonar ou cardiovascular, comprometimento ortopédico ou neuromuscular que limitassem a realização do teste.

\subsection{Procedimentos}

Os participantes foram submetidos a realizar o teste de 2400 metros para a determinação do $\mathrm{VO}_{2}$ máx, sendo realizado uma única vez na pista de corrida do Parque Potycabana. Antes e após o teste foi realizado a avaliação dos sinais vitais de pressão arterial (PA) através do aparelho de pressão, frequência cardíaca (FC) pela palpação manual da artéria radial, saturação (SPO2) através do oxímetro e frequência respiratória (FR) por meio da observação.

Os materiais utilizados foram o esfigmomanômetro (Premium) e estetoscópio (Premium) e o oxímetro de pulso (Fingertip Pulse), após a corrida foi feita uma nova avaliação com apuração dos sinais vitais após a corrida.

A obtenção dos dados no teste de 2400 metros (12 minutos), consiste em avaliar quanto tempo os atletas percorreram a distância prevista, e se caso houver ultrapassagem dos 12 minutos, utilizar de cálculos para as informações adequadas como subsídio da pesquisa que foi realizada.

\subsection{Análise Estatística}

Os procedimentos estatísticos foram realizados no Software Statistical Package for the Social Sciences-SPSS, versão 22.

A análise estatística consta de descrição das frequências das variáveis e associação (volume de oxigênio máximo (VO2 máx), modalidade esportiva, aptidão física, idade, sexo e tempo de treinamento). 
Nas análises de associação o $\mathrm{VO}_{2}$ será considerado como variável dependente. Será considerado nível significante de $5 \%(p<0,05)$ para rejeição da hipótese de igualdade.

\section{Resultados e Discussão}

Dos 14 voluntários que atenderam aos critérios de inclusão do estudo 10 participantes sexo masculino $(71,4 \%)$ e 4 sexo feminino (28,6\%), 8 voluntários de futsal (57,1\%) e 6 de handebol (42,9\%). Foi utilizado o teste de associação Qui-quadrado de associação dos dados qualitativos sociais (idade, sexo e modalidade esportiva), foi também utilizado o teste de comparação de média para os dados quantitativos para os resultados fisiológicos (frequência de treinamento, pressão arterial média inicial, pressão arterial média final, frequência cardíaca, máx e tempo de percurso). Tabela 1

Tabela 1 - Análise Descritiva dos dados Sociais e Fisiológicos de estudantes universitários de um Centro Universitário Particular da capital. Teresina (PI)-2019.N:14

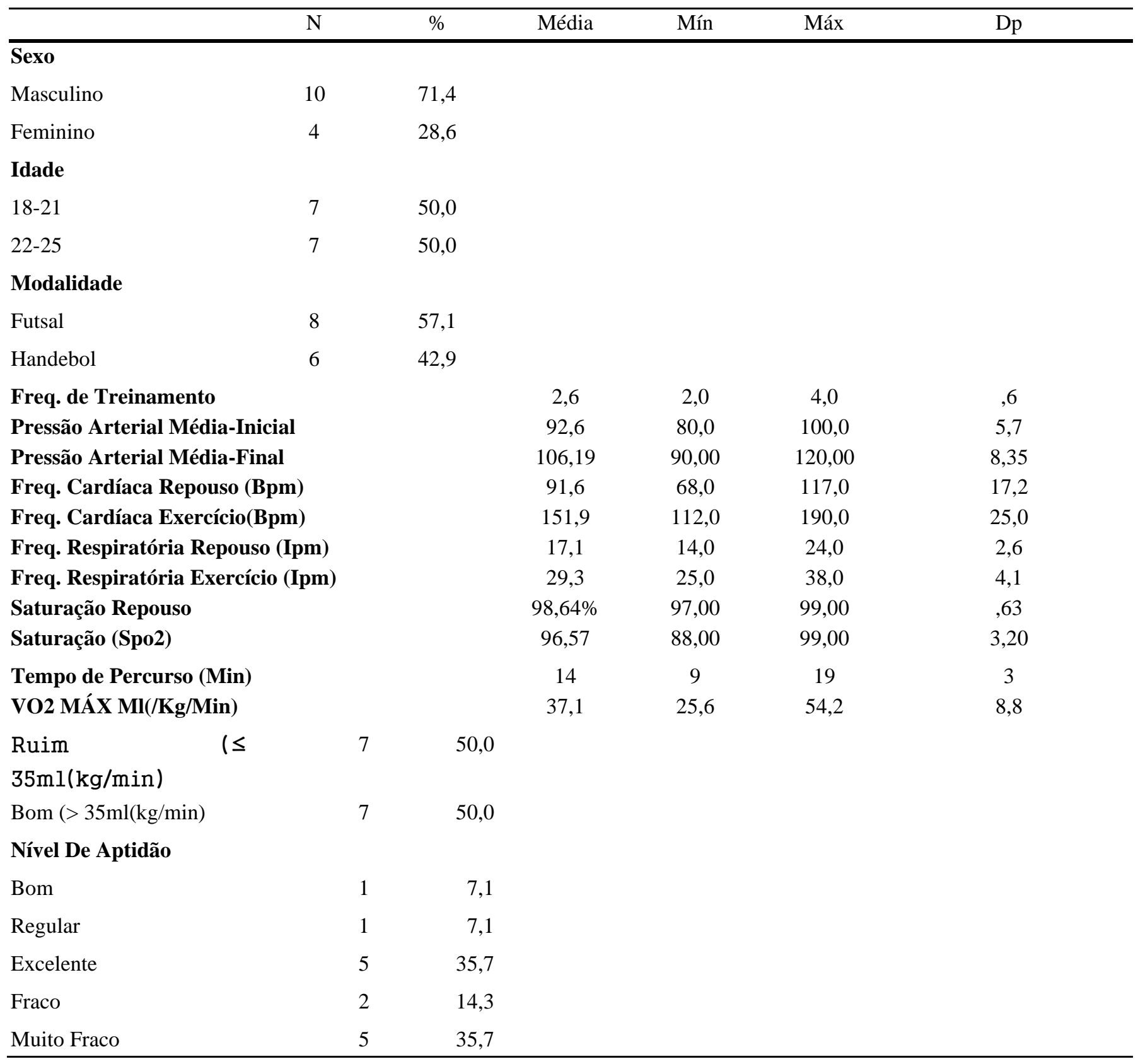

Fonte: Autores, Teresina Pi, (2019). 
Com base no estudo o teste de associação (Qui-quadrado) em nível de significância de 5\% (p< 0,05) à evidencias estatísticas existindo associação entre o VO2máx entre sexo e nível de aptidão, porém em comparação com idade e modalidade não houve evidências pois não existiu P-valor que mostre valor abaixo de 5\%.

Os dados Fisiológicos quantitativos dos estudantes universitários mostrou que valores abaixo de 5\% demonstraram que a sequência de valores não segue a distribuição normal, ou seja, os valores acima de $5 \%$ à evidências estatísticas com base no teste na Tabela 2

Tabela 2 - Análise Inferencial dos dados Sociais e Fisiológicos de estudantes universitários de um Centro Universitário Particular da capital em relação ao VO2máx ml(kg/min). Teresina (PI)-2019.N:14

\begin{tabular}{|c|c|c|c|c|c|}
\hline & \multicolumn{4}{|c|}{ VO2 MÁX ml(/kg/min) } & \multirow[b]{3}{*}{ P-valor } \\
\hline & \multicolumn{2}{|c|}{$\operatorname{Ruim}(\leq 35 \mathrm{ml}(\mathrm{kg} / \mathrm{min})$} & \multicolumn{2}{|c|}{ Bom $(>35 \mathrm{ml}(\mathrm{kg} / \mathrm{min})$} & \\
\hline & $\mathrm{N}$ & $\%$ & $\mathrm{~N}$ & $\%$ & \\
\hline$\overline{\text { Sexo }}$ & & & & & $0,018^{* *}$ \\
\hline Masculino & 7 & 50,0 & 3 & 21,4 & \\
\hline Feminino & 0 & 0,0 & 4 & 28,6 & \\
\hline Idade & & & & & $0,109^{*}$ \\
\hline $18-21$ & 2 & 14,3 & 5 & 35,7 & \\
\hline $22-25$ & 5 & 35,7 & 2 & 14,3 & \\
\hline Modalidade & & & & & $0,28^{*}$ \\
\hline Futsal & 5 & 35,7 & 3 & 21,4 & \\
\hline Handebol & 2 & 14,3 & 4 & 28,6 & \\
\hline NÍVEL DE APTIDÃO & & & & & $0,007 * *$ \\
\hline Bom & 0 & 0,0 & 1 & 7,1 & \\
\hline Regular & 0 & 0,0 & 1 & 7,1 & \\
\hline Excelente & 0 & 0,0 & 5 & 35,7 & \\
\hline Fraco & 2 & 14,3 & 0 & 0,0 & \\
\hline Muito Fraco & 5 & 35,7 & 0 & 0,0 & \\
\hline
\end{tabular}

Fonte: Autores, Teresina Pi (2019).

De acordo com o teste de normalidade (teste de Shapiro-Wilk) dos dados fisiológicos quantitativos dos estudantes universitários, mostrou que valores abaixo de 5\% demonstram que a sequência de valores não segue a distribuição normal, ou seja os valores acima de $5 \%$ à evidências estatísticas com base no teste. Tabela 3 
Tabela 3 -Teste de normalidade dos dados fisiológicos quantitativos de estudantes universitários de um Centro Universitário Particular da capital em Teresina (PI)-2019.N:14.

\begin{tabular}{lccc} 
& \multicolumn{3}{c}{ Shapiro-Wilk } \\
\cline { 2 - 4 } & Estatística & Df & P-valor \\
\hline Freq. de Treinamento & 0,77 & 14 & $\mathbf{0 , 0 0 2}$ \\
Pressão Arterial Média-Inicial & 0,91 & 14 & 0,181 \\
Pressão Arterial Média-Final & 0,97 & 14 & 0,849 \\
Freq. Cardíaca Repouso (Bpm) & 0,90 & 14 & 0,110 \\
Freq. Cardíaca Exercício (Bpm) & 0,94 & 14 & 0,481 \\
Freq. Respiratória Repouso (Ipm) & 0,82 & 14 & $\mathbf{0 , 0 0 8}$ \\
Freq. Respiratória Exercício (Ipm) & 0,77 & 14 & $\mathbf{0 , 0 0 2}$ \\
Saturação(Spo2) Repouso & 0,63 & 14 & $\mathbf{0 , 0 0 0}$ \\
Saturação (Spo2) & 0,76 & 14 & $\mathbf{0 , 0 0 2}$ \\
Tempo de Percurso (Min) & 0,94 & 14 & 0,366 \\
\hline
\end{tabular}

Fonte: Autores, Teresina Pi (2019).

*Teste de Normalidade Shapiro-Wilk, ao nível de significa de 5\%

Conforme os dados obtidos na Tabela 4 o teste de comparação (T De Student), a pressão arterial apresentou p- valor de 0,001 e tempo de repouso apresentou p-valor de 0,00 mostrando evidências estatísticas entre as diferenças dos grupos bom e ruim. Porém a pressão arterial média inicial, pressão arterial média final, frequência cardíaca, frequência cardíaca pós-corrida, frequência respiratória pós-corrida, e tempo de percurso, não houve evidências estatísticas entre esses grupos, ou seja, tanto grupo de VO2máx bom e ruim não obtiveram diferenças, apresentando o p-valor $<0,05$ entre pressão arterial final, tempo de repouso e frequência de treinamento. Os dados estão representados na Tabela 4

Tabela 4 - Análise Inferencial dos dados fisiológicos quantitativos de estudantes universitários de um Centro Universitário Particular da capital em relação ao VO2máx ml(kg/min). Teresina (PI)-2019. N:14.

\begin{tabular}{|c|c|c|c|c|c|}
\hline & \multicolumn{4}{|c|}{ VO2 MÁX ml(/kg/min) } & \multirow[b]{4}{*}{ P-valor } \\
\hline & \multirow{2}{*}{\multicolumn{2}{|c|}{$\begin{array}{c}\text { Ruim ( } \leq 35 \mathrm{ml} \\
(\mathrm{kg} / \mathrm{min})\end{array}$}} & \multirow{2}{*}{\multicolumn{2}{|c|}{ Bom $(>35 \mathrm{ml}(\mathrm{kg} / \mathrm{min})$}} & \\
\hline & & & & & \\
\hline & Média & $\mathrm{Dp}$ & Média & $\mathrm{Dp}$ & \\
\hline Freq. de Treinamento & 2,3 & ,5 & 3,0 & 6 & $0,053 * *$ \\
\hline Pressão Arterial Média-Inicial & 92,4 & 6,6 & 92,9 & 5,1 & $0,8733^{*}$ \\
\hline Pressão Arterial Média-Final & 112,39 & 5,67 & 100,00 & 5,44 & $0,001 *$ \\
\hline Freq. Cardíaca Repouso (Bpm) & 96,6 & 19,7 & 86,7 & 14,1 & $0,302 *$ \\
\hline Freq. Cardíaca Exercício (Bpm) & 158,1 & 25,7 & 145,6 & 24,4 & $0,367^{*}$ \\
\hline Freq. Respiratória Repouso (Ipm) & 16,1 &, 7 & 18,0 & 3,5 & $0,456^{* *}$ \\
\hline Freq. Respiratória Exercício (Ipm) & 29,1 & 4,1 & 29,4 & 4,4 & $0,902 * *$ \\
\hline Saturação Repouso & $99,00 \%$ & $0,00 \%$ & $98,29 \%$ &, $76 \%$ & $0,073 * *$ \\
\hline Saturação (Spo2) & $97,00 \%$ & $2,31 \%$ & $96,14 \%$ & $4,06 \%$ & $0,902 * *$ \\
\hline Tempo de Percurso (Min) & 17 & 1 & 11 & 1 & $0,00^{*}$ \\
\hline
\end{tabular}

Fonte: Autores, Teresina Pi (2019).

*Teste de Comparação de grupos não pareados, de Teste T De Student, ao nível de significa de 5\%

***Teste de Comparação de grupos não pareados, de Teste U de Mann Whitney ao nível de significa de 5\%. 
O VO2 máx. vem sendo considerado um dos parâmetros de grande importância como preditor de performance, pois a capacidade do ser humano para realizar exercícios de longa e média duração depende principalmente do metabolismo aeróbio sendo, assim, um índice muito empregado para classificar a capacidade funcional cardiorrespiratória, sobretudo em atletas (Cecil, 2017).

O presente estudo objetivou avaliar o VO2máx entre 14 atletas, de diferentes modalidades esportivas, acadêmicas de uma Instituição de Ensino Superior da capital do Piauí, o Centro Universitário Santo Agostinho, sendo 8 atletas de futebol e 6 atletas de handebol de sexos variados.

Nossos dados presentes na Tabela 1, mostram uma média de VO2máx entre todos os atletas coletados de 37, 1 ml/kg/min- 1, caracterizando VO2máx com um bom nível de aptidão física.

Verificou-se a presença significante das variáveis pertencentes à aptidão física, que na Tabela 2 relaciona-se com o VO2máx bom e ruim, demonstrado também por (Araújo, 2017) quando em sua pesquisa avaliando 86 militares do sexo masculino, observou na corrida de $50 \mathrm{~m}$, a velocidade como aptidão física e de maneira geral obtiveram melhora significativa entre o início e o final da avaliação, e ainda ressalta que a aptidão física é importante para o estado de prontidão.

As exposições fisiológicas: pressão arterial média final e tempo de percurso, expressos na Tabela 3 e 4, mostram nível de $\mathrm{p}>5 \%$ e $\mathrm{p}<5 \%$, respectivamente, em comparação com o VO2 bom e ruim, verificando assim que essas variáveis possuem diferenças significantes no teste de normalidade e análise inferencial.

(Souza, 2016) avaliou em 48 atletas e dividiu-os em 4 grupos com 12 atletas em cada, sendo o último grupo avaliando atletas de handebol, $50 \%$ dos atletas que coletamos tiveram idade entre 18-21 anos e 50\% tiveram idade entre $22-25$ como exemplifica a presença dessas idades no estudo de Souza et al. fora seu primeiro grupo que teve atletas abaixo de 18 anos, apresentando um melhor desempenho muscular e do nível de oxigênio máximo entre os atletas de futebol em comparação aos de handebol.

O proposto por esse estudo compara modalidade e sexo expresso pela Tabela 1 que mostra a presença de $28,7 \%$ de mulheres no total, o handebol como integrante as 4 mulheres presentes, resultado da aptidão: melhor resultado handebol do que o com atletas futebol, mas sendo insignificante pela presença de p $>5 \%$, pelo sexo sim com uma significância de $p<5 \%$, contrapondo assim a pesquisa de (Sousa, 2018) que mostra as mulheres com um VO2 máx inferior aos homens.

No estudo de Mahseredjan,1999) 63 jogadores de futebol de campo, sexo masculino, com idade variando de 15 à 20 anos, com média de VO2máx de 59,77 ml/kg/min, encontrados por meio do teste de Cooper foram de $54,49 \mathrm{ml} / \mathrm{kg} / \mathrm{min}-1$, gerando um VO2 máximo bom comparado com nossas tabelas que exemplificam VO2 máx ( $<35 \mathrm{ml} / \mathrm{kg} / \mathrm{min}-1$, ruim; >35 $\mathrm{ml} / \mathrm{kg} / \mathrm{min}-1$, bom), tendo como próprio resultado $\mathrm{r}=0,23$ não significante para a amostra de 58 atletas.

(Triani, 2018) pesquisou 16 estudantes de Educação Física de uma faculdade particular localizada no Rio de Janeiro, de ambos os sexos, executou 3 testes durante 3 dias com intervalo de 48h, para valores de VO2máx foi utilizado Bleep Fitnen Test, o qual consiste na demarcação de um percurso totalizando 20m, gerando média de VO2máx 35,33 ml/kg/min-1 de acordo com a nossas tabelas também possuindo um VO2 máximo bom.

\section{Considerações Finais}

Os resultados mostraram que existe nível de significância entre VO2 máx, aptidão física, sexo, frequência cardíaca média final e tempo de percurso, presente nos 14 atletas avaliados. Não houve resultados significativos entre as variáveis: modalidade, em comparação ao nível de VO2máx e idade. Faz-se de suma importância a pesquisa do presente estudo pela escassez de trabalhos publicados sobre o tema, engrandecendo assim o artigo proposto para o meio acadêmico, científico e literário. 
Sendo assim a necessidade de projetos para corroborar com os dados dispostos, contudo ressalta a importância de adentrar as questões relacionadas às variáveis idade e volume de oxigênio máximo com estudos mais aprofundados sobre o assunto.

\section{Referências}

Alves, J. C., Perisseli, A. C., \& Nogueira, G. A. (2015). Comparação entre os métodos direto e indireto de determinação do VO2máx de praticante de corrida, Rev. brasileira de medicina e esporte, 21(1)

Araújo, L., \& Moura, G. M. (2017). Aptidão física e lesões: 54 semanas de treinamento físico com policiais militares. Revista Brasileira de Medicina do Esporte, $23(2)$

Baganha, R. J, Dias, R., Cieslak, F., \& Camarço, N. F. (2017). Parâmetros Imunológicos E Infecções Do Trato Respiratório Superior Em Atletas De Esportes Coletivos, 23(1) Rev. Brasileira de Medicina do Esporte.

Bortoloti, R., Rodrigues, N. C., \& Cortez, M. D. (2013). Overtraining increases the strength of equivalence relations. Psychology \& Neuroscience, 6(3).

Cecil, F., Lima,T., Mineiro, A., \& Guedes, D. P. (2017). Características fisiológicas e antropométricas de atletas profissionais de futebol e futsal. Rev. brasileira de futsal e futebol, $9(32)$.

Corrêa, B. D., Pereira, R. N., Lira, D., Avila, P. S., Moreno, A., \& Normando, V., (2018). Avaliação e classificação da capacidade física aeróbia de atletas de basquetebol em cadeira de rodas. Revista (Brasileira de Ciências do Esporte, 40 (2), 163-169).

Freitas, V., Bara, M. G., \& Miloski. B. (2014). Quantificação da carga de treinamento através do método percepção subjetiva do esforço da sessão e desempenho no futsal. Rev. bras. Cineantropom e desempenho humano 14 (1):73-82.

Junior, E. C., Souza, F. B., \& Martins, R. A.(2015) Estudo comparativo do consumo de oxigênio e limiar anaeróbio em um teste de esforço progressivo entre atletas profissionais de futebol e futsal. Rev Bras Med Esporte; 12 (6):323-6.

Mahseredjian, F., Barros,N., Tebexreni, L. T., \& Sérgio, A., (1999). Estudo comparativo de métodos para a predição do consumo máximo de oxigênio e limiar anaeróbio em atletas. Revista Brasileira de Medicina do Esporte, 5 (5).

Moreira, P., \& Rizza, C. V. (2014). Concordância entre dois testes de capacidade aeróbica máxima baseadas nas corridas de esteiras e pista, com jogadores de futsal, Rev. da Estatísticas da Universidade Federal de Ouro Preto, (2).

Triani, D. F., (2018). Correlação Entre Índice de Massa Corporal, Potência Muscular e Consumo de Oxigênio de Estudantes de Educação Física. Journal of Health Sciences, 20(1).

Pereira, E. N., (2017) Assessment of Central Blood Pressure and Arterial Stiffness in Practicing Long-Distance Walking Race. International Journal of Cardiovascular Sciences.

Politano, H., Politano, F. N., Silva, A. P. S., Pereira, A. D. A., \& Pellegrinotti, Í. L. (2020). Estudo comparativo da potência aeróbica entre os estágios maturacionais determinados pela menarca. Revista de Salud Pública, (22)

Rangel, G. O., Júnio, A. C., \& Marcelo, B. A. (2018). Prevalência de lesões em atletas universitários praticantes de futebol e futsal, Rev. brasileira de futebol e futsal, 10(41).

Reis, D. F., Júnior, M. L., \& Rotta, H. I. (2018). Análise do consumo máximo de oxigênio e da composição corporal em atletas de futebol da categoria sub-20, Rev. Colloquium Vitae,(10).

Rodrigues, A. N., Perez, A. J., Carletti, L., Bissoli, N., \& Abreu, G. (2007). Aptidão cardiorrespiratória e associações com fatores de riscos cardiovascular em adolescente, Jornal de Pediatria (83)5: 429-435

Souza, K. M., Vieira, G., \& Baldi, M. F. (2016). Variáveis fisiológicas neuromusculares associadas com a performance aeróbica em corredores de endurece: Efeitos da distância da prova, Rev. brasileira de medicina e esporte, (17)1.

Sousa, F. D. B., Ferreira, R. C. A., Fernandes, W. S., Ribeiro, W., \& Lazo-Osorio, R. A. (2018). Comparison of aerobic power and capacity between athletes from different sports. Revista Brasileira de Medicina do Esporte, (24) 6: 432-435).

Spigolon, L. M. P., Cavaglieri, C. R., Gomes, A. C., \& Pascoal, E. H. (2017). Training program influences the relation between functional and neuromuscular performance indicators during the season in young soccer players. Rev. Brasileira de Ciências do Esporte, (39)1 : 98-106.

Kellmann, M., Bertollo., M., \&Bosquet L.(2018). Recovery and performance in sport: consensus statement. International journal of sports physiology and performance,(13) $2: 240-245$. 